\title{
Kinematics of the Ionized Gas in Dwarf Irregular Galaxies
}

\author{
Margarita Valdez-Gutiérrez ${ }^{1}$ and Margarita Rosado ${ }^{2}$ \\ ${ }^{1}$ Instituto de Astronomía - Universidad Nacional Autónoma de México, campus Ensenada, \\ Ensenada, B. C., Mexico \\ ${ }^{2}$ Instituto de Astronomía - Universidad Nacional Autónoma de México, Mexico City, Mexico
}

\begin{abstract}
Some preliminary results focused on the kinematics and dynamics of the ionized gas in the Local Group dwarf irregular galaxy IC10 are presented.
\end{abstract}

Keywords. galaxies: dwarf, galaxies: irregular, ISM: kinematics and dynamics, instrumentation: interferometers

\section{Results and Discussion}

The results here presented are part of a long term project intended to study the interaction between massive stars and the interstellar medium in Local Group dwarf irregular galaxies (Valdez-Gutiérrez, Rosado, Georgiev et al. (2001), Rosado, ValdezGutiérrez, Georgiev et al. (2001), Valdez-Gutiérrez, Rosado, Puerari et al. (2002)). This proceeding is devoted to IC10 which is unique among other dwarf irregulars due to its starburst classification. The observations were carried out by means of PUMA, a scanning Fabry-Perot interferometer, attached to the f/7.9 Ritchey-Chrétien focus of the $2.1 \mathrm{~m}$ telescope at the Observatorio Astronómico Nacional. Data cube acquisition was performed in the $\mathrm{H} \alpha$, [SII], [NII] and [OIII] lines. The dedicated ADHOC package was used in the reduction process. From the moment maps we find that on galactic scales the radial velocity field in this galaxy is relatively well behaved with a bit complex kinematics at the south of the giant HII complex HL111 (Hodge \& Lee (1990)); due to this reason the rotation curve has been difficult to obtain. On local scales the kinematics reflects the superposition of shells, filaments, SNRs and diffuse gas, particularly at the location where a non thermal superbubble has been reported. At many of these locations the velocity widths are supersonic and many times larger (a factor of 2-3) than those found in the adjacent HII regions (velocity dispersions $\sim 20 \mathrm{~km} / \mathrm{s}$ ). From these preliminary results a very complex behavior of the ionized gas is unveiled, as has been the case for the dwarf irregulars IC1613 and NGC4449, previously studied by our group. Further analysis is being carried out (Valdez-Gutiérrez et al. 2007, in preparation).

\section{References}

Hodge, P. \& Lee, M. G. 1990, PASP 102, 26

Rosado, M., Valdez-Gutiérrez, M., Georgiev, L. et al. 2001, AJ 122, 194

Valdez-Gutiérrez, M., Rosado, M., Georgiev, L. et al. 2001, A\&SA 366, 35

Valdez-Gutiérrez, M., Rosado, M., Puerari, I. et al. 2002, AJ 124, 3157 